http://jmscr.igmpublication.org/home/

ISSN (e)-2347-176x ISSN (p) 2455-0450

crossref DOI: https://dx.doi.org/10.18535/jmscr/v7i7.116

\title{
Diagnosis and Treatment of Superior Mesenteric Artery Syndrome: Our Experience in a Tertiary Hospital
}

\author{
Authors \\ Dr Manmadha Rao Vayalapalli ${ }^{1}$, Dr Chaithanya Babu Bogarapu ${ }^{2 *}$, Dr Prudhvi Raj \\ Karumuri $^{3}$, Dr Aditya Ram Munagavalasav ${ }^{4}$, Dr Satya Niharika Vadisetti ${ }^{5}$ \\ ${ }^{1}$ Professor of Surgery, ${ }^{2}$ Assistant Professor of Surgery, ${ }^{3,4,5}$ Post graduate in Surgery \\ Andhra Medical College and King George Hospital, Visakhapatnam, Andhra Pradesh, INDIA - 530001 \\ *Corresponding Author \\ Dr Chaithanya Babu Bogarapu
}

Assistant Professor of Surgery, Andhra Medical College, Visakhapatnam, Andhra Pradesh - 530001, India

\section{Abstract}

Superior mesenteric artery (SMA) syndrome is an uncommon cause of gastrointestinal tract obstruction which is accompanied with epigastric pain and vomiting. Normally, the angle created by the aorta and the SMA is between 45 - 60 degrees. Any factor that narrows this aorto-mesenteric angle to less than 25 degrees can result in entrapment and compression of the third part of the duodenum and leading to the SMA syndrome.

This paper describes our experience in the diagnosis and management of 5 cases of this rare entity in our tertiary care hospital.

Keywords: Superior Mesenteric Artery Syndrome, SMA, narrow aorto-mesenteric angle, Mega duodenum.

\section{Introduction}

Superior mesenteric artery (SMA) syndrome is an uncommon cause of gastrointestinal tract obstruction which presents great diagnostic challenge to the treating surgeon and the patient may be often misdiagnosed leading to lifethreatening complications. Superior mesenteric artery (SMA) syndrome was first described by an Austrian Professor Carl Freiherr von Rokitansky in his anatomy text $1842^{[1]}$ and subsequently, Wilkie published the comprehensive series of 75 patients in $1927^{[2]}$ and later analyzed by Barner and Sherman ${ }^{[3]}$. throughout the literature, SMA syndrome has been variably referred to as Cast syndrome, Wilkie syndrome, arterio-mesenteric duodenal obstruction, and chronic duodenal ileus ${ }^{[4]}$.

The underlying pathology is by external compression between the 3rd and fourth part of the duodenum between the SMA and aorta. The narrowing of the aorto-mesenteric angle results in a decrease in distance between the aorta and the SMA with consequent compression of the intervening segment of the duodenum. The mean angle between the SMA and the abdominal aorta is approximately $45^{\circ}$ (range of $38-56^{\circ}$ ). Precipitating factors that narrow the aortomesenteric angle by approximately $6-25^{\circ}$ can cause this syndrome ${ }^{[5]}$. SMA syndrome is extremely rare, evident in only $0.3 \%$ of upper gastrointestinal tract barium studies ${ }^{[6]}$. The true incidence of this syndrome is unknown, but it has 
been estimated to be approximately 0.013 $0.3 \%{ }^{[7]}$.

Thin body habitus may predispose to SMA syndrome. Also, catabolic states and malnutrition, rapid weight reduction by reduction in mesenteric and retroperitoneal fat tissue can predispose to SMA syndrome. Tuberculosis ${ }^{[8]}$, diabetes mellitus ${ }^{[9]}$, anorexia nervosa ${ }^{[10]}$, blunt abdominal trauma $^{[11]}$, and burns ${ }^{[12]}$ are among the many described causes of SMA syndrome. All patients requiring prolonged bed rest following severe injury are at risk. SMA syndrome may also occur following corrective spinal surgery ${ }^{[13]}$ and bariatric surgeries ${ }^{[14]}$

\section{Materials and Methods}

We have managed six cases of intestinal obstruction due to SMA syndrome during the time period of June 2015 to January 2019. The study was conducted in a tertiary care hospital of North Coastal Andhra Pradesh, Visakhapatnam in India. Our catchment area includes the districts of North Andhra, Orissa and even parts of Jharkhand and West Bengal. Records were analyzed with special focus on presenting complaints, clinical findings, investigations done, the treatment offered and post-operative period.

\section{Results}

Of the six cases presented to us 2 were males and 4 were females. The ages of the cases range from 13 years to 26 years with an average of 20.5 years.

Ages distribution of presenting cases

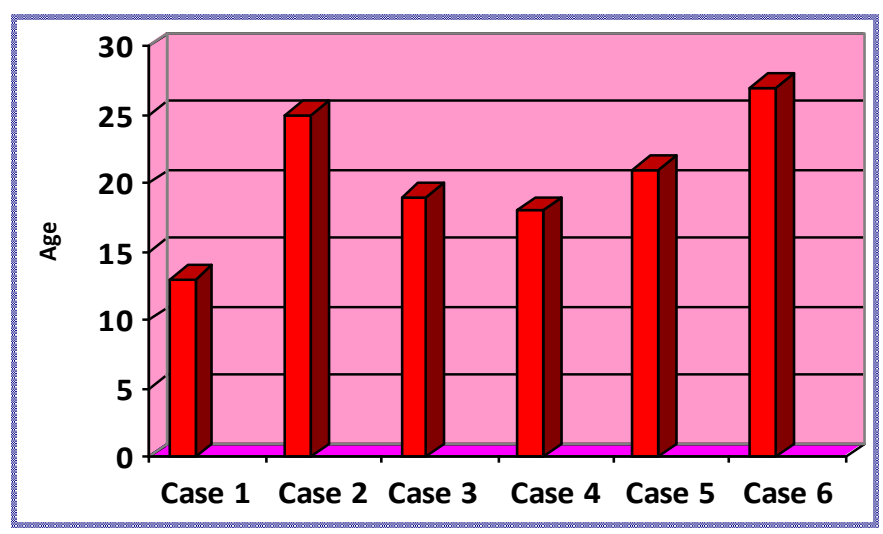

The common presenting symptoms were vomiting in 6 cases $(100 \%)$, nausea in 4 cases (67\%), vague abdominal pain or discomfort in 4 cases $(67 \%)$, relief of symptoms with positional changes in 2 cases (33\%), and rapid weight loss prior to surgery/management in 3 cases $(50 \%)$.

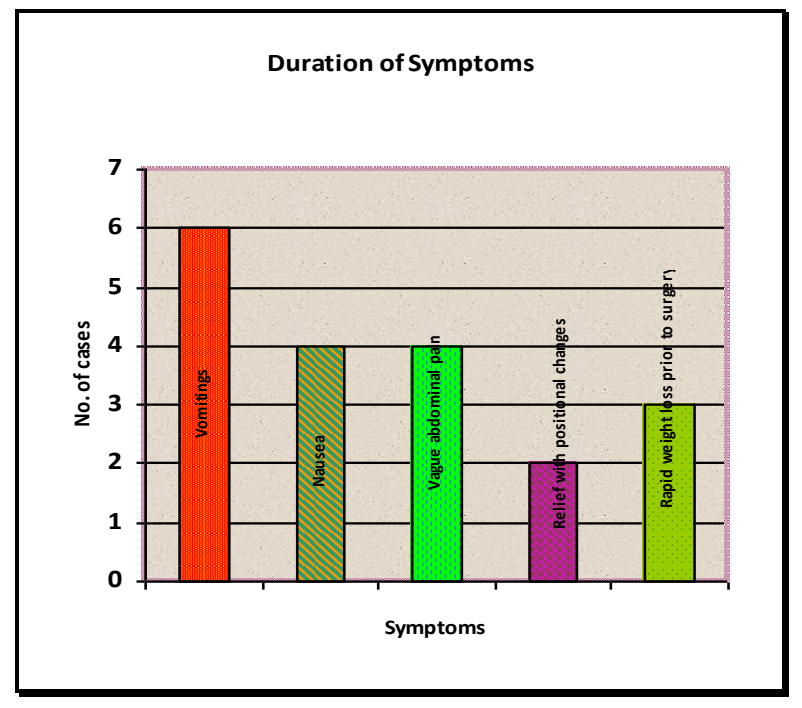

The presenting symptoms duration ranged from 4 months to 72 months with a mean duration of symptoms of 25.6 months.

Upper Gastro-intestine endoscopy and CECT were performed on all these six cases. All cases showed dilated duodenum except in one case where the duodenum was normal. In one case, apart from the dilated duodenum, there was also a stricture at the $3^{\text {rd }}$ part of the duodenum due to extrinsic compression on endoscopy.

In all the cases the aorto-mesenteric angle is less than the normal range of $38-56^{\circ}$ on CECT. The aorto-mesenteric angle of the six cases ranged from $18^{\circ}$ to $23^{\circ}$ with a mean of $20.5^{\circ}$.




Surgery was performed on all the six cases. All procedures were done by open method. Access was gained by upper Mid-line laparotomy in 4 cases, right sub costal approach in 1 case, and by right transverse approach in 1 case.

All the cases had dilated proximal duodenum with constriction between the third and fourth part of the duodenum. One case had stricture between the third and the fourth part of the duodenum. The same case also had calcified mesenteric lymph nodes signifying tuberculosis as the causative agent. All cases showed a narrow aorto-mesenteric angle.

Duodeno-jejunostomy was performed in 4 cases, duodeno-duodenostomy in 1 case, Roux-en-y jejunostomy in 1 case, and Ligament of Trietz lysis (Strong's procedure) in 1 case.

Post operatively 1 case developed enterocutaneous fistula which was re-explored and resection and side-to-side jejuno-duodenal anastomosis was done. Four cases developed vomiting for 2 weeks which were managed by Erythromycin.

\section{Discussion}

Superior mesenteric artery syndrome has many eponyms such as chronic duodenal ileus, megaduodenum, aortomesenteric artery compression, arteriomesenteric duodenal obstruction, cast syndrome, and chronic duodenal pseudoobstruction $^{[15]}$.

The syndrome is routinely seen in young females who present with signs and symptoms of acute or chronic intestinal obstruction. Most patients of SMA syndrome give a history of vague upper abdominal pain with or without vomiting after intake of food, associated with upper abdominal fullness and belching. The cases are generally treated as intestinal obstruction and managed in the same lines when presenting in acute form. More commonly these patients present with chronic vomiting of unexplained etiology. Danger of labeling such cases as functional cases is always there.

In acute presentation resuscitation with NG tube decompression and IV fluids should be started. Barium swallow will show dilatation of proximal duodenum and stomach. An ultrasound will reveal reduced aorto-mesenteric angle and distance with proximal dilatation. But the definitive diagnosis can be made on a CECT abdomen with cuts in the sagittal section showing the compression of duodenum by SMA.

The SMA takes off from the abdominal aorta at the level of the first lumbar vertebra with an average angle of $45^{\circ}$ (range $18^{\circ}$ to $70^{\circ}$ ) and a distance of $10-28 \mathrm{~mm}$ suspended by the ligament of Treitz, which is attached to its 4th part or to its junction with the jejunum, the duodenum crosses the abdomen at the level of the third lumbar vertebra. A narrow aorto- mesenteric angle of $15.2^{\circ}$ (range $1^{\circ}-40^{\circ}$ ) and a narrow aortomesenteric distance of 2 to $8 \mathrm{~mm}$ have been observed in individuals with SMA syndrome ${ }^{[16]}$.



Normal and Narrow Aorto-mesenteric angle

Thinning out of the fat pad between SMA and aorta due to rapid weight loss, narrows the aortomesenteric angle and distance, and compresses the duodenum producing the clinical manifestations of the syndrome. Other contributory factors include an abnormally low origin of SMA, excessive lumbar lordosis, and hypertrophied or shortened ligament of Treitz or its multiple attachments to the duodenum ${ }^{[16]}$. High fixation of the duodenum by the ligament of Treitz or an anomalous SMA crossing directly over the aorta as the it transects the duodenum has also 
been identified as a cause ${ }^{[17]}$.

An upper GI endoscopy is routinely done for a chronic case of SMA syndrome reveals narrowing of the 3rd part of the duodenum due to external compression.

Once diagnosed a trial of medical management by decompression of the stomach and duodenum with a nasogastric tube, correction of nutritional and electrolytes deficiencies by fluids and TPN can be tried. Positional change in form of right recumbent positioning after intake of food also relieves compression in few cases and helps relieve obstruction.

Failure of medical management, surgical options can be tried. Side-to-side duodeno-jejunostomy is by far the most successful treatment for SMA syndrome. Gastrojejunostomy can also performed in some cases. Lysis of ligament of trietz with mobilization of the second and third part of the duodenum, the so called Strong's procedure can be done as an add on procedure or a standalone procedure $^{[18]}$. Post operatively these patients may need prokinetics for a short duration as most of the cases suffered from persistent vomiting.

\section{Conclusion}

SMA syndrome is an established cause of intestinal obstruction. But diagnosing this condition needs a high degree of suspicion. The diagnosis is rather one of exclusion than straightforward. Radiological investigations such as CECT abdomen help in definitively diagnosing the condition. Simple procedure in form of side to side duodeno-jejunostomy can provide great relief to the patient and the surgeon must be aware of this condition before labelling the patient as a functional.

\section{Source of support or grant: NIL}

\section{References}

1. Rokitansky C: Handbuch der pathologischen Anatomie, ed 1. Wien, Braunmuller \& Seidel 1842, vol 3, p187.

2. Wilkie DPD: Chronic duodenal ileus. Am
J Med Sci 1927;173:643-649.

3. Barner HB, Sherman CD., Jr Vascular compression of the Duodenum. Int Abstr Surg. 1963 Aug;117:103-118.

4. Welsch T, Buchler MW, Kienle P: Recalling Superior Mesenteric Artery Syndrome. Dig Surg. 2007; 24(3):149-56

5. Agrawal GA, Johnson PT, Fishman EK: Multidetector row CT of superior mesenteric artery syndrome. J Clin Gastroenterol. 2007 Jan;41(1):62-5.

6. Lowell S. Goin, Stefan P. Wilk. Intermittent Arteriomesenteric Occulusion of the Duodenum. Radiology (Nov 1, 1956) 67(5):729-737.

7. Ylinen P, Kinnunen J, Hockerstedt K. J Clin Gastroenterol. 1989 Aug; 11(4):38691.

8. C. S. Limaye, S. P. Karande, S. P. Aher, and K. A. Pati, "Superior mesenteric artery syndrome secondary to tuberculosis induced cachexia," The Journal of the Association of Physicians of India, vol. 59, pp. 670-671, 2011.

9. M.-C. Wu, I.-C. Wu, J.-Y. Wu, D.-C. Wu, and W.-M. Wang, "Superior mesenteric artery syndrome in a diabetic patient with acute weight loss," World Journal of Gastroenterology, vol. 15, no. 47, pp. 6004-6006, 2009.

10. C. W. Lee, M. I. Park, S. J. Park et al., “A case of superior mesenteric artery syndrome caused by anorexia nervosa," The Korean Journal of Gastroenterology, vol. 58, no. 5, pp. 280-283, 2011.

11. J. L. Falcone and K. O. Garrett, "Superior mesenteric artery syndrome after blunt abdominal trauma: a case report," Vascular and Endovascular Surgery, vol. 44, no. 5, pp. 410-412, 2010.

12. T. J. Lescher, K. R. Sirinek, and B. A. Pruitt Jr., "Superior mesenteric artery syndrome in thermally injured patients," Journal of Trauma, vol. 19, no. 8, pp. 567571,1979 
13. Altiok H, Lubicky JP, DeWald CJ, Herman JE: The superior mesenteric artery syndrome in patients with spinal deformity. Spine 2005;30:2164-2170.

14. D. Goitein, D. J. Gagné, P. K. Papasavas et al., "Superior mesenteric artery syndrome after laparoscopic Roux-en-Y gastric bypass for morbid obesity," Obesity Surgery, 2004 vol. 14, no. 7, pp. 10081011.

15. M. Sturtevant, "Megaduodenum and duodenal obstruction. Criteria for diagnosis," Radiology, vol. 33, no. 2, pp. 185-188, 1939.

16. C. M. Townsend and J. J. Naoum, "Vascular compression of the duodenum," in Mastery of Surgery, J. E. Fischer, Ed., pp. 955-961, Lippincott Williams \& Wilkins, 5th edition, 2007.

17. L. B. Cohen, S. P. Field, and D. B. Sachar, "The superior mesenteric artery syndrome. The disease that isn't, or is it?" Journal of Clinical Gastroenterology, vol. 7, no. 2, pp. 113-116, 1985.

18. W. Z. Massoud, "Laparoscopic management of superior mesenteric artery syndrome," International Surgery, vol. 80, no. 4, pp. 322-327, 1995. 